\title{
Pure Spinal Epidural Cavernous Hemangioma with Intralesional Hemorrhage: A Rare Cause of Thoracic Myelopathy
}

\author{
Donghwan Jang ${ }^{1}$, Choonghyo Kim¹, Seung Jin Lee', Young-Joon Ryu², Jiha Kim \\ Departments of ${ }^{l}$ Neurosurgery, ${ }^{2}$ Pathology, Kangwon National University Hospital, Chuncheon, Gangwon, Korea
}

\begin{abstract}
Although cavernous hemangiomas occur frequently in the intracranial structures, they are rare in the spine. Most of spinal hemangiomas are vertebral origin and "pure" epidural hemangiomas not originating from the vertebral bone are very rare. Our spinal hemangioma case is extremely rare because of its "pure" epidural involvement and intralesional hemorrhage. A 64-year-old man presented with progressive paraparesis from two months ago. His motor weakness was rated as grade $4 / 5$ in bilateral lower extremities. He also complained of decreased sensation below the T4 sensory dermatome, which continuously progressed to the higher dermatome level. Magnetic resonance imaging demonstrated thoracic spinal tumor at T3-T4 level. The tumor was located epidural space compressing thoracic spinal cord ventrally. The tumor was not involved with the thoracic vertebral bone. We performed T3-5 laminectomy and removed the tumor completely. The tumor was not infiltrating into intradural space or vertebral bone. The histopathologic study confirmed the epidural tumor as cavernous hemangioma. Postoperatively, his weakness improved gradually. Four months later, his paraparesis recovered completely. Here, we present a case of pure spinal epidural cavernous hemangioma, which has intralesional hemorrhage. We believe cavernous hemangioma should be included in the differential diagnosis of the spinal epidural tumors.
\end{abstract}

Key Words: Cavernous $\cdot$ Epidural $\cdot$ Hemangioma $\cdot$ Hemorrhage $\cdot$ Spine

\section{INTRODUCTION}

Pure spinal epidural cavernous hemangiomas (PSECHs) are extremely rare ${ }^{4,7,10)}$. Although cavernous hemangiomas appear frequently in the intracranial structures, they are rare in the spine $^{1-3,6,10)}$. About $80 \%$ of the cavernous hemangiomas are supratentorial, $15 \%$ infratentorial and only $5 \%$ are located in the spine ${ }^{10)}$. However, most of spinal hemangiomas are vertebral origin with or without epidural space extension ${ }^{2,3,5,12,13)}$. Most of the epidural hemangiomas are secondary extensions from the vertebral lesion and the "pure" epidural hemangiomas not originating from the vertebral bone are very rare ${ }^{1,5,9,13)}$. The review of the literature has revealed only 80 reported cases of PSECH till date ${ }^{1)}$.

Therefore, little is known about its natural course and clin-

- Received: April 28, 2014 - Revised: June 5, 2014

- Accepted: June 20, 2014

Corresponding Author: Jiha Kim, MD

Department of Neurosurgery, Kangwon National University Hospital, Baengnyeong-ro, 156, Chuncheon-si, Gangwon-do 200-722, Korea Tel: +82-33-258-2410, Fax: +82-33-251-0374

E-mail: dopaa@naver.com

®This is an Open Access article distributed under the terms of the Creative Commons Attribution Non-Commercial License (http://creativecommons.org/ licenses/by-nc/3.0/) which permits unrestricted non-commercial use, distribution, and reproduction in any medium, provided the original work is properly cited. ical characteristics. The paucity of information stem from its rarity and lack of literature. Most knowledge about PSECH is derived from several sporadic case reports and large series studies are very limited ${ }^{3}$.

Here, we present a case of PSECH, which has intralesional hemorrhage. Our case is extremely rare because of its "pure" epidural involvement and intralesional hemorrhage. We believe our case can be useful addition to existing knowledge about the PSECHs.

\section{CASE REPORT}

A 64-year-old man presented to the outpatient clinic with progressive paraparesis which had begun two months ago.

His bilateral lower extremity weakness was rated as grade 4/5 in bilateral hip and knee joints. He had difficulty in walking independently and he frequently slipped down during walking by himself. He also complained of decreased sensation below the T4 sensory dermatome. Although his back pain was tolerable, the hypesthesia continuously progressed to the higher sensory dermatome level. Deep tendon reflexes of the knee and ankle produced normal finding. And he did not have any problem in urinary function.

Magnetic resonance imaging (MRI) demonstrated thoracic spinal tumor at T3-4 level. The tumor was located dorsal epi- 
dural space and was compressing thoracic spinal cord ventrally. There was no epidural hemorrhage surrounding the tumor and the tumor was not involved with any part of the thoracic vertebral bone (Fig. 1).

We performed an operation to remove tumor with total laminectomy in the prone position. After removal of T3-5 laminas and ligament flavum the epidural tumor was exposed. The tumor was dark-red color and well demarcated from peripheral venous plexus. There was no epidural hematoma surrounding the tumor and dural adhesion was minimal. Although there was some bleeding, the tumor was totally removed. We opened the dural membrane minimally and checked there was no intradural infiltration. The tumor was not involved with the nerve root or vertebral bone. The histopathologic study confirmed the epidural tumor as cavernous hemangioma (Fig. 2). There was no postoperative complication.

Immediate after the operation, his lower extremity weakness showed no change. However, his neurological deficit improved gradually. Four months later, follow-up visits revealed normal strength in both lower extremities, and he was able to walk independently at this time.

\section{DISCUSSION}

Cavernous hemangiomas are composed of dilated sinusoidal capillaries lined by a single layer of benign endothelial cells ${ }^{6,13)}$, which are separated by variably thin fibrous adventitia but not by neural parenchyma ${ }^{14}$.

According to International Society for the Study of Vascular Anomalies (ISSVA) classification, vascular anomalies were classified into two subgroups: neoplasms and malformations ${ }^{14)}$. "Hemangioma" is characterized by tumor-like endothelial proliferation and spontaneous involution; it refers to infantile or congenital hemangioma in this classification. However, PSECHs differ from "hemangioma" of infancy, which is benign vascular tumor that usually regresses spontaneously ${ }^{4)}$. Moreover, there is no evidence that PSECHs may grow by mitotic activity ${ }^{6}$. Caruso et al. ${ }^{2)}$ mentioned that the specific epidural localization may be explained by the embryological development of dura mater; the vascular elements from the primordial plexus may have some influence in the postnatal development of a cavernomatous lesion. Thus, PSECHs are congenital or developmental vascular malformations and are clearly differentiated from vascular neoplasms ${ }^{3,4,6,7,10)}$. Vascular malformations are classified by the predominant type of vascular channel (i.e. capillary, cavernous, arteriovenous, or venous) ${ }^{3,7,11)}$. Cavernous hemangioma is composed of dilated sinusoidal vascular channels, differential from capillary-sized vessels in capillary hemangioma ${ }^{13)}$.
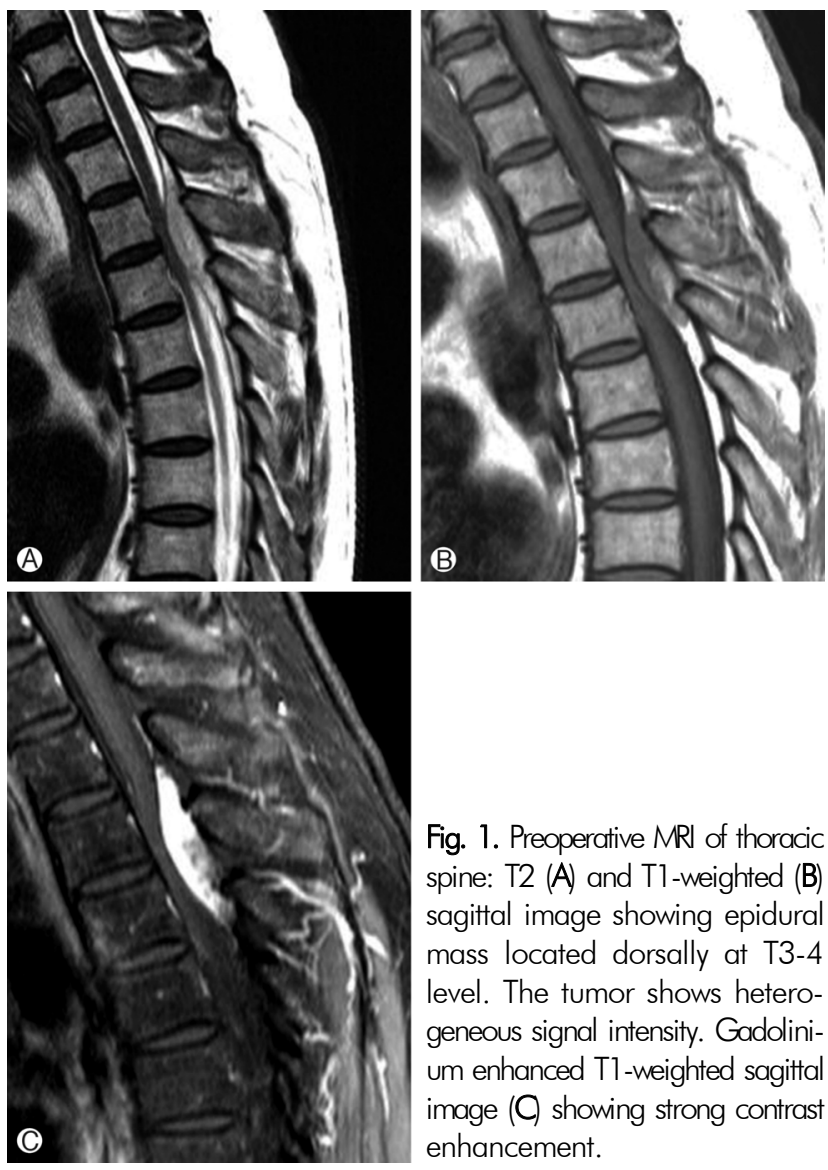

Fig. 1. Preoperative MRl of thoracic spine: T2 (A) and T1-weighted (B) sagittal image showing epidural mass located dorsally at T3-4 level. The tumor shows heterogeneous signal intensity. Gadolinium enhanced Tl-weighted sagittal image (C) showing strong contrast enhancement.
On histopathologic examination, our case was composed of a large number of dilated vascular channels walled by thin endothelial cells. The prominent venous lakes and dilated vascular channels, mainly of the cavernous type, were filled with abundant of red blood cells (Fig. 2A). Although the tumor showed strong positive in cluster of differentiation (CD) 34 staining (Fig. 2B), it was negative on CD31 staining (Fig. 2C), which implies that there was no lymphatic component in our tumor. Thus, we confirmed the tumor was cavernous hemangioma without lympangioma component.

Although PSECHs do not represent true neoplasms, they are dynamic lesions, and intralesional hemorrhage, thrombosis, organization, cyst formation and involution of the caverns, all contribute to the changes in size and nature of these lesions, with subsequent spinal cord compression, necessitating complete surgical removal as the treatment of choice, ${ }^{3,4,6}$.

Hemorrhages are common in intra-axial cavernous hemangiomas but less frequent in extra-axial ones ${ }^{2,10)}$. Although spontaneous spinal epidural hemorrhage is rare and account for less than $1 \%$ of all spinal epidural lesions, the most common causes include coagulopathies, hypertension, increased venous pressure and vascular malformation ${ }^{5)}$. However, intralesional 

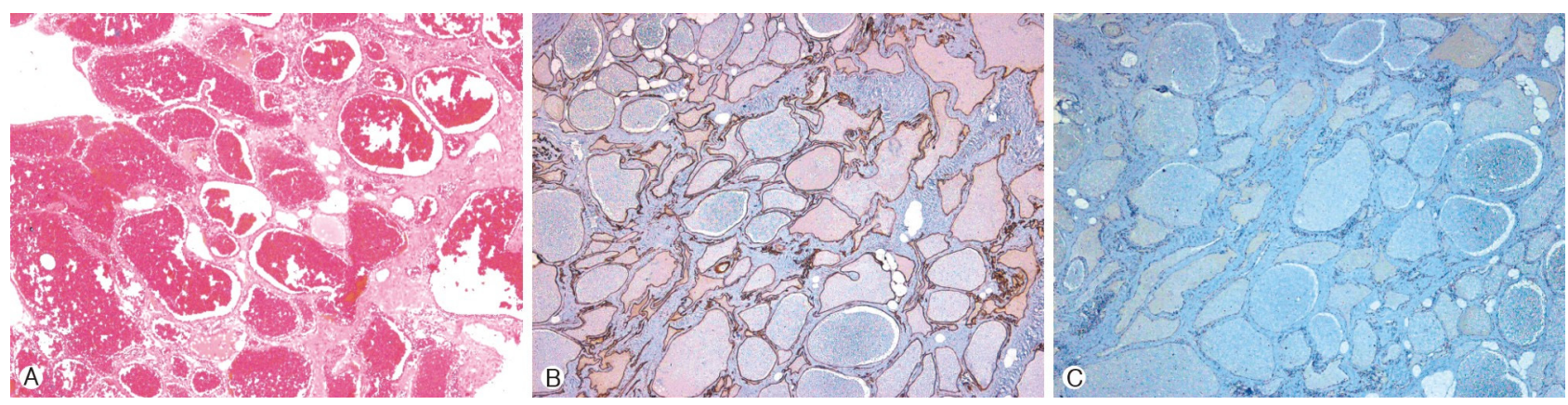

Fig. 2. Histopathologic examination: (A) A microscopic view of mass. The tumor was composed of prominent venous lakes and dilated sinusoidal capillaries walled by a single layer of endothelial cell, characterizing a cavernous hemangioma (Hematoxyline and Eosin staining, original magnification X40). Immunohistochemical examination showed positive staining for CD34 (B), but negative staining of the endothelial cells for CD31 (C), implying the absence of lympangioma component (original magnification X40)

hemorrhage without epidural hematoma is rare and is reported sporadically in only limited literature, related with acute symptom aggravation ${ }^{4,6}$.

Common clinical presentation of PSECH is a progressive myelopathy or radiculopathy, in some patients back pain is the only clinical sign of the lesion ${ }^{6,9,13)}$. Acute onset of symptoms, especially with a very significant neurological deficit is rare ${ }^{2,4,5,9,10)}$. And it can be explained by a sudden increase in volume of the lesion, mainly due to hemorrhage, or thrombosis of the draining veins ${ }^{5,6,8,9)}$. Although intramedullary cavernomas show progressive myelopathy followed by acute deterioration, the protective effect of the dura and cerebrospinal fluid may play a role in preventing spinal cord injury in $\mathrm{PSECH}^{2}$. However, there are some reports about acute neurologic symptom induced by acute hemorrhage ${ }^{2,4-7)}$. We think insidious symptom onset in our case stems from repeated intralesional microhemorrhages rather than acute massive tumor bleeding. In our case, there was no obvious epidural hemorrhage into the surrounding tissue. We confirmed the absence of epidural hematoma on preoperative MRI and at the time of surgery.

According to literature, PSECHs showed high T2 signal intensity and homogeneous, strong contrast enhancement on $\mathrm{MRI}^{3,5-7,9)}$. This pattern is different from the intra-axial cavernomas, which do not enhance homogeneously ${ }^{2}$. Although our case demonstrated strong contrast enhancement, heterogeneous signal intensity on T1- and T2-weighted MRI were clues of the intralesional hemorrhage, which was confirmed intraoperatively. Heterogeneous enhancement pattern after recurrent bleeding has been reported by some authors ${ }^{4}$. Although the intramedullary or intracerebral cavernous hemangiomas frequently show a peripheral low signal intensity rim, representing hemosiderin deposition from recurrent intralesional hemorrhage, PSECHs usually do not show a low signal rim like our case ${ }^{2,5,13)}$. This is presumably caused by the easier removal of blood products outside the blood-brain barrier ${ }^{5}$.
They are most commonly located in the posterior aspect of thoracic spine as in our case ${ }^{3,4,6,8)}$. It is thought to be related with several factors; the larger available epidural space, ${ }^{3,6}$, the lower resistance in the posterior portion of the spinal canal ${ }^{6}$, and the abundant venous plexus in the dorsal or dorsolateral of the epidural space ${ }^{13)}$.

Despite the advancement of MRI technique, PSECHs are usually misdiagnosed as other lesions. The PSECH should be differentiated from other epidural, neoplastic or inflammatory lesions, such as meningioma, lymphoma, metastasis, and hemorrhagic vascular lesions ${ }^{5,13}$. Although they are less frequently found in the foraminal and extraforaminal regions ${ }^{6,12)}$, schwannoma or neurinoma should also be considered as differential diagnosis.

Because of the high vascularization of hemangiomas, preoperative misinterpretation may result in unexpected intraoperative hemorrhage and incomplete resection, which results in the persistence of clinical symptoms or recurrence ${ }^{3,7}$. Some authors recommended en-bloc removal to avoid intraoperative blood loss, ${ }^{2,13)}$, which is possible only after accurate preoperative diagnosis. Although bleeding is seldom a problem with intramedullary cavernous hemangiomas during surgery, severe intraoperative bleeding may occur in spinal epidural lesions ${ }^{6}$. Reoperation for remnant or recurrent epidural hemangioma is very difficult because of peridural or periradicular adhesion and unclear tumor margins; as a result, complete resection cannot be guaranteed in reoperation ${ }^{7}$. Therefore, proper preoperative planning and complete resection in the first operation is essential ${ }^{7}$.

In summary, we believe that the accurate preoperative diagnosis and complete resection is indispensable to favorable treatment outcome. And PSECHs should be considered as differential diagnosis for spinal epidural lesions. 


\section{CONCLUSION}

Pure epidural cavernous hemangiomas with intralesional hemorrhage are very rare finding in spinal region. However, the hemangioma should be included in the differential diagnosis of spinal epidural tumors, even though it has intralesional hemorrhage.

\section{REFERENCES}

1. A LH, T R, Chamarthy NP, Puri K: A pure epidural spinal cavernous hemangioma - with an innocuous face but a perilous behaviour!! J Clin Diagn Res 7:1434-1435, 2013

2. Caruso G, Galarza M, Borghesi I, Pozzati E, Vitale M: Acute presentation of spinal epidural cavernous angiomas: case report. Neurosurgery 60:E575-576; discussion E576, 2007

3. Feng J, Xu YK, Li L, Yang RM, Ye XH, Zhang N, et al: MRI diagnosis and preoperative evaluation for pure epidural cavernous hemangiomas. Neuroradiology 51:741-747, 2009

4. Floeth F, Riemenschneider M, Herdmann J: Intralesional hemorrhage and thrombosis without rupture in a pure spinal epidural cavernous angioma: a rare cause of acute lumbal radiculopathy. Eur Spine J 19:S193-196, 2010

5. Jo BJ, Lee SH, Chung SE, Paeng SS, Kim HS, Yoon SW, et al: Pure epidural cavernous hemangioma of the cervical spine that presented with an acute sensory deficit caused by hemorrhage.

Yonsei Med J 47:877-880, 2006

6. Khalatbari MR, Abbassioun K, Amirjmshidi A: Solitary spinal epidural cavernous angioma: report of nine surgically treated cases and review of the literature. Eur Spine J 22:542-547, 2013

7. Lee JW, Cho EY, Hong SH, Chung HW, Kim JH, Chang KH, et al: Spinal epidural hemangiomas: various types of MR imaging features with histopathologic correlation. AJNR Am J Neuroradiol 28:1242-1248, 2007

8. Sanghvi D, Munshi M, Kulkarni B, Kumar A: Dorsal spinal epidural cavernous hemangioma. J Craniovertebr Junction Spine 1:122-125, 2010

9. Saracen A, Kotwica Z: Thoracic spinal epidural cavernous haemangioma with an acute onset: Case report and the review of the literature. Clin Neurol Neurosurg 115:799-801, 2013

10. Satpathy DK, Das S, Das BS: Spinal epidural cavernous hemangioma with myelopathy: a rare lesion. Neurol India 57:8890, 2009

11. Son S, Lee SG, Yoo CJ, Park CW, Kim WK: Pure Thoracic Spinal Epidural Cavernous Hemangioma with Spinal Cord Compression: A Case Report. Korean J Spine 7:116-119, 2010

12. Uchida K, Yayama T, Nakajima H, Hirai T, Kobayashi S, Chen $\mathrm{K}$, et al: Microsurgical resection of cavernous haemangioma around the thoracic neuroforamen: a case report. J Orthop Surg (Hong Kong) 18:370-373, 2010

13. Zhong W, Huang S, Chen H, Sun H, Cai B, Liu Y, et al: Pure spinal epidural cavernous hemangioma. Acta Neurochir (Wien) 154:739-745, 2012

14. Zhong W, You C: Spinal cavernous hemangioma: a controversial terminology. Acta Neurochir (Wien) 154:1251-1252, 2012 